\title{
Low-Dose Spironolactone-Pioglitazone-Metformin Normalizes Circulating Fetuin-A Concentrations in Adolescent Girls with Polycystic Ovary Syndrome
}

\author{
Marta Díaz, ${ }^{1,2}$ José Miguel Gallego-Escuredo, ${ }^{3,4,5}$ Abel López-Bermejo, ${ }^{6}$ Francis de Zegher, \\ Francesc Villarroya, ${ }^{1,3,4}$ and Lourdes Ibáñez $\mathbb{D}^{1,2}$ \\ ${ }^{1}$ Endocrinology, Institut Pediàtric Hospital Sant Joan de Déu, University of Barcelona, Esplugues, 08950 Barcelona, Spain \\ ${ }^{2}$ Centro de Investigación Biomédica en Red de Diabetes y Enfermedades Metabólicas Asociadas (CIBERDEM), ISCIII, Madrid, Spain \\ ${ }^{3}$ Department of Biochemistry and Molecular Biology, and Institute of Biomedicine, University of Barcelona, 08028 Barcelona, Spain \\ ${ }^{4}$ Centro de Investigación Biomédica en Red Fisiopatología de la Obesidad y Nutrición (CIBEROBN), ISCIII, Madrid, Spain \\ ${ }^{5}$ Institut de Recerca Biomèdica de Lleida, Lleida, Spain \\ ${ }^{6}$ Department of Pediatrics, Dr. Josep Trueta Hospital, and Girona Institute for Biomedical Research, 17007 Girona, Spain \\ ${ }^{7}$ Department of Development and Regeneration, University of Leuven, Leuven, Belgium
}

Correspondence should be addressed to Lourdes Ibáñez; libanez@hsjdbcn.org

Received 21 September 2017; Accepted 20 December 2017; Published 19 July 2018

Academic Editor: Antonio Simone Laganà

Copyright (c) 2018 Marta Díaz et al. This is an open access article distributed under the Creative Commons Attribution License, which permits unrestricted use, distribution, and reproduction in any medium, provided the original work is properly cited.

Background. Fetuin-A is a glycoprotein produced in the liver and related to metabolic syndrome; fetuin-A secretion is divergently regulated in different pathological conditions. In girls with polycystic ovary syndrome (PCOS), insulin sensitization results in a more favorable endocrine-metabolic outcome than oral contraception; we assessed whether those differences are underscored by changes in circulating fetuin-A. Methods. Fetuin-A concentration endocrine-metabolic markers and hepatovisceral fat were measured longitudinally in 35 PCOS girls [age, $16 \mathrm{yr}$; body mass index (BMI), $23 \mathrm{~kg} / \mathrm{m}^{2}$ ] randomized to receive either oral contraception [ethinylestradiol-levonorgestrel $(n=18)$ ] or a low-dose combination of spironolactone, pioglitazone, and metformin (SPIOMET, $n=17$ ) over 12 months. Healthy adolescent girls (age- and BMI-matched) were used as controls $(n=25)$. Results. Pretreatment fetuin-A serum levels in PCOS girls were lower than those in controls. After 12 months on treatment, fetuinA raised to control levels only in the SPIOMET subgroup $(P=0.009$, versus oral contraception); this increase was paralleled by a healthier metabolic profile with less hepatic fat (by MRI); baseline serum fetuin-A as well as the changes over 12 months was inversely related to hepatic adiposity. Conclusions. A low-dose combination of insulin sensitizers and an antiandrogen-but not oral contraception—normalizes fetuin-A levels in adolescent girls with PCOS. This trial is registered with ISRCTN29234515.

\section{Introduction}

Fetuin-A ( $\alpha 2$-HS glycoprotein, AHSG) is a glycoprotein produced primarily in the liver and secreted into circulation in high concentrations in humans with fatty liver disease [1]; it binds the insulin receptor and inhibits hepatic and muscle insulin signaling resulting in insulin resistance [2]. In humans, high levels of fetuin-A have been associated with greater risks for type 2 diabetes (T2D) and with features of the metabolic syndrome [3]; paradoxically, increased
fetuin-A concentrations prevent vascular calcification and exert a protective role in systemic inflammation, suggesting that fetuin-A secretion can be divergently regulated in different pathological conditions [4].

Polycystic ovary syndrome (PCOS) is the most common cause of hirsutism, acne, and menstrual irregularity in girls and young women and associates to comorbidities in adulthood, including subfertility and T2D. We have previously shown that in adolescent girls with PCOS, oral contraception (OC) - as compared to combined low-dose insulin 
sensitization plus antiandrogen therapy-is linked to a less favorable endocrine-metabolic profile $[5,6]$. To date, the available studies reporting fetuin-A in PCOS have been performed in adult women, include heterogeneous populations, and have a cross-sectional nature [7-9]. Here, we assessed longitudinally whether the divergent effects of oral contraception and low-dose combined insulin sensitization plus antiandrogen therapy in adolescent PCOS are underscored by changes in circulating fetuin-A.

\section{Subjects and Methods}

2.1. Study Population and Design. The study population consisted of 35 nonobese adolescent girls with PCOS [mean age, $16 \mathrm{yr}$; body mass index (BMI), $\left.23 \mathrm{~kg} / \mathrm{m}^{2}\right]$; all of them were at least $2 \mathrm{yr}$ beyond menarche. The inclusion criteria were as described [10] (1) hirsutism (score $>8$ on Ferriman and Gallwey scale); (2) oligomenorrhea (menstrual intervals $>45$ days); and (3) absence of sexual activity throughout the study duration (and thus, no need for contraception). The girls were recruited in the Adolescent Endocrinology Unit of Sant Joan de Déu University Hospital, Barcelona, Spain [10]. Recruitment was biased against overweight/obesity because, in our setting, those girls are primarily referred to the adolescent obesity unit. Exclusion criteria were thyroid dysfunction, anemia, bleeding disorder, hyperprolactinemia, Cushing syndrome, adrenal hyperplasia, liver or kidney dysfunction, and use of drugs affecting gonadal or renal function or carbohydrate or lipid metabolism. Patients were included in a randomized, open-label study comparing the effects of OC with ethinylestradiol-levonorgestrel [EE-LNG; $20 \mu \mathrm{g}$ of EE plus $100 \mathrm{mg}$ of LNG for 21 of 28 days, placebo for 7 of 28 days; Loette Diario, Pfizer, Madrid, Spain] with those of a low-dose combination of spironolactone $50 \mathrm{mg} / \mathrm{d}$, pioglitazone $7.5 \mathrm{mg} / \mathrm{d}$, and metformin $850 \mathrm{mg} / \mathrm{d}$ (SPIOMET). The study was registered as ISRCTN29234515 and lasted for 24 months (12 months on treatment; then 12 months off treatment) [10]. The present report specifically included those girls with complete longitudinal data in whom the remaining serum sample was sufficiently abundant to measure fetuin-A at baseline and after 12 months on treatment ( $90 \%$ of the initial study population, $n=18$ and $n=17$ in the EE-LNG and SPIOMET subgroups, resp.). Twenty-five age- and BMI-matched healthy girls recruited in nearby schools served as controls. All had regular menstrual cycles, and none was hirsute or was on OC or other medications affecting ovarian function or carbohydrate or lipid metabolism.

2.2. Clinical and Endocrine-Metabolic Assessments. One investigator (unblinded to treatment) measured weight and height (Harpenden Stadiometer) and scored hirsutism (FerrimanGallwey). Systolic and diastolic blood pressures were recorded after a 5-minute rest with the girl supine, using an electronic sphygmomanometer (767 series, Welch Allyn, Spain).

Endocrine-metabolic assessments were performed in the early morning, in the follicular phase (days 3-7) of the cycle or after 2 months of amenorrhea, as described [5]. Briefly, circulating insulin and SHBG were assayed by immunochemiluminescence (IMMULITE 2000, Diagnostic
Products, Los Angeles, CA). HOMA-insulin resistance (HOMA-IR) was calculated as [fasting insulin in $\mathrm{mU} / \mathrm{L}] \times$ [fasting glucose in $\mathrm{mg} / \mathrm{dL}] / 405$. Serum C-reactive protein (CRP) was analyzed by immunochemiluminescence (ARCHITECT i2000SR, Abbott Diagnostics, Abbot Park, IL, USA); intra- and interassay coefficients of variation (CVs) were $<10 \%$. HMW adiponectin was assessed by ELISA (R\&D Systems, Minneapolis, MN, USA); intra- and interassay CV s were $<9 \%$. Circulating fetuin-A was assessed with a specific ELISA (fetuin-A, R\&D systems, Minneapolis, $\mathrm{MN}, \mathrm{USA}$ ); the intra- and interassay CVs were $4.2 \%$ and $7.4 \%$, respectively.

2.3. Abdominal Fat Partitioning. Subcutaneous, visceral, and hepatic fat was assessed by magnetic resonance imaging (MRI) using a multiple-slice MRI 1.5 Tesla scan (Signa LX Echo Speed Plus Excite, General Electric, Milwaukee, WI) [10].

2.4. Statistical Analyses and Ethics. Statistical analyses were performed with SPSS 23.0 (SPSS Inc. Chicago, IL). Results are expressed as mean \pm SEM. Comparisons within and between groups at each time point were performed using general linear model. Correlation analysis was used to study the associations between fetuin-A levels and auxological and endocrine-metabolic parameters. Two-way analysis of variance (ANOVA) was performed to assess the influence of treatment and time on fetuin-A levels. $P<0.05$ was considered statistically significant.

The study was conducted after approval by the Institutional Review Board of Sant Joan de Déu University Hospital, after written consent by parents and after assent by each of the participants, including the healthy controls who allowed to obtain indicative values.

\section{Results}

Both treatments reduced androgen excess comparably, but SPIOMET was followed by a more favorable endocrinemetabolic profile, as expected (see Table 1 for differences in selected variables between subgroups).

Pretreatment serum concentrations of fetuin-A in PCOS girls were lower than those in controls. After 12 months on treatment, fetuin-A levels increased only in the SPIOMET subgroup ( $P=0.009$ versus the OC subgroup), reaching control levels (Figure 1, Table 1).

At baseline, circulating fetuin-A correlated negatively with hepatic fat in both controls and PCOS girls $(r=-0.739$; $P=0.03$ and $r=-0.446 ; P=0.006$, resp.). After treatment, fetuin-A negatively associated with visceral fat in the SPIOMET subgroup $(r=-0.583, P=0.004)$ and with diastolic blood pressure in both the SPIOMET and OC subgroups ( $r=-0.729, P=0.002$, and $r=-0.584, P=0.03$, resp.). The change in serum fetuin-A concentrations $0-12$ months correlated negatively with diastolic blood pressure $(r=-0.442 ; P=0.039)$, hepatic fat $(r=-0.647 ; P=0.002)$, and C-reactive protein (CRP; $r=-0-617 ; P=0.003$ ), only in the SPIOMET subgroup. Two-way ANOVA showed that both time and treatment have an effect on fetuin-A levels $(P=0.02$ and $P=0.006$, resp.). 
TABLE 1: Data from adolescent girls with polycystic ovary syndrome (PCOS) who were randomized to receive either ethinylestradiollevonorgestrel (EE-LNG; $n=18$ ) or low-dose spironolactone-pioglitazone-metformin (SPIOMET; $n=17$ ) for 12 months.

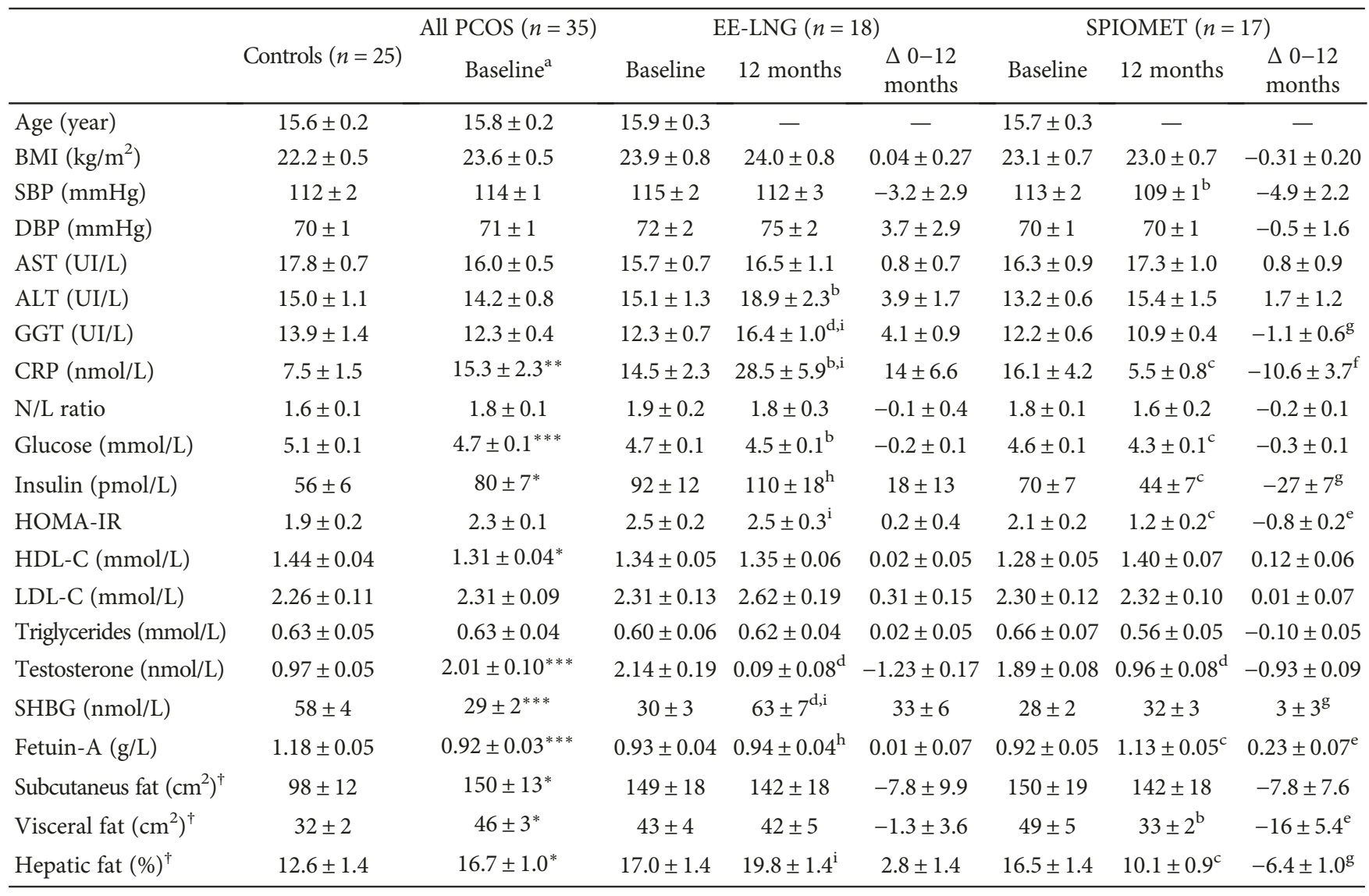

Values are mean \pm SEM. BMI: body mass index; SBP: systolic blood pressure; DBP: diastolic blood pressure; CRP: c-reactive protein; N/L: neutrophile-to-lymphocyte ratio; HOMA-IR: homeostatic model assessment-insulin resistance; HMW: adiponectin, high molecular weight; TC: total cholesterol; TG, triglycerides; SHBG: sex hormone binding globulin; D4-A: 4-androstenedione; DHEAS: dehydroisoandrosterone sulphate. ${ }^{\dagger}$ By MRI; ${ }^{*} p<0.05,{ }^{* *} p<0.01$, and ${ }^{* * *} p<0.001$ between controls and PCOS girls at baseline. ${ }^{\mathrm{a}}$ No significant differences between randomized PCOS subgroups at baseline; ${ }^{\mathrm{b}} p<0.05,{ }^{\mathrm{c}} p<0.01$, and ${ }^{\mathrm{d}} p<0.001$ within subgroups for 0 -to-12-month changes $(\Delta) ;{ }^{\mathrm{e}} p<0.05,{ }^{\mathrm{f}} p<0.01$, and ${ }^{\mathrm{g}} p<0.001$ between subgroups for 0 -to-12-month changes $(\Delta)$; ${ }^{\mathrm{h}} p<0.01$ and ${ }^{\mathrm{i}} p<0.001$ between subgroups at 12 months.

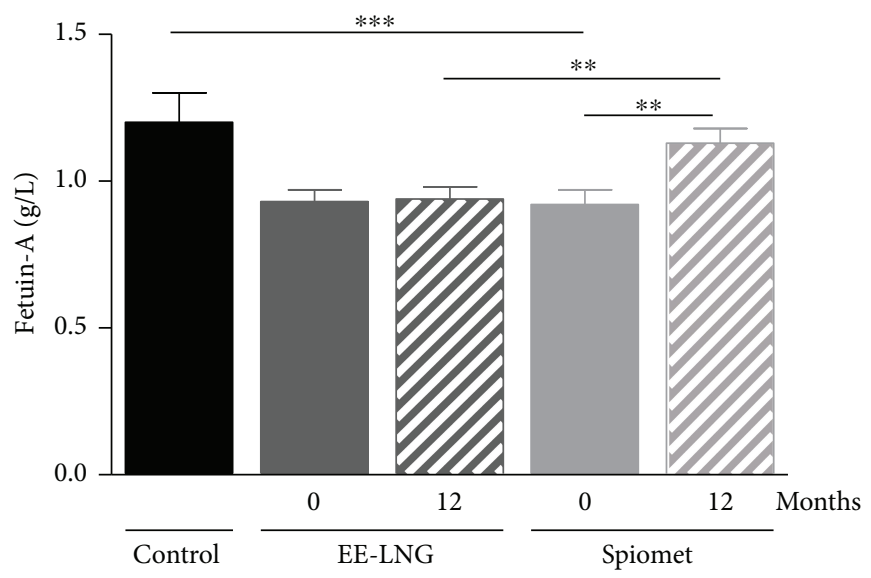

FIGURE 1: Longitudinal fetuin-A concentrations (mean \pm SEM) in adolescent girls with polycystic ovary syndrome who were randomized to receive either an oral contraceptive [ethinylestradiol-levonorgestrel (EE-LNG) $n=18$ ] or low-dose spironolactone (50 mg/d), pioglitazone $(7.5 \mathrm{mg} / \mathrm{d})$ plus metformin $(850 \mathrm{mg} / \mathrm{d})$ (SPIOMET, $n=17)$, for 12 months. Control girls $(n=25)$ matched for age and body mass index were assessed for comparison. ${ }^{* *} P<0.001$ for baseline differences between patients and controls; ${ }^{* *} P<0.01$ within patient subgroups for 0 to 12 months and between groups at 12 months. 


\section{Discussion}

Here, we characterize for the first time the longitudinal outcome of circulating fetuin-A concentrations in nonobese adolescents with PCOS and show that fetuin-A levels normalize after treatment with a low-dose combination of insulin sensitizers and an antiandrogen, but not after OC.

Growing evidence supports the relationship between fetuin-A and hepatic fat depots in obesity [11]. Also, strong associations between fetuin- $\mathrm{A}$ and insulin resistance have been reported in subjects without diabetes [12]; indeed, fetuin-A promotes insulin resistance through inhibition of insulin receptor tyrosine kinase in hepatocytes and skeletal muscle and activation of Toll-like receptor 4 in response to free fatty acids, stimulating an inflammatory signaling pathway $[2,13]$. In contrast, other studies report no associations between fetuin- $\mathrm{A}$ and insulin resistance in T2D patients or suggest that fetuin-A is not causally related to diabetes risk [14, 15]. Moreover, the impact of fetuin-A on cardiovascular disease is controversial depending on the presence or absence of diabetes. For example, nondiabetic subjects-but not T2D patients-with higher fetuinA levels have a lesser risk for cardiovascular disease and related mortality [14]. Besides, fetuin-A would prevent liver and vascular fibrosis through the inhibition of transforming growth factor- $\beta 1$ signaling [16].

Our apparently discordant data could be partially explained bearing in mind that fetuin-A comprises a mixture of circulating isoforms regulating specific biological processes and that the available ELISA assays may differ in their specificity for different glycosylated forms [17]. Moreover, defects of glycosylation/sialylation of fetuin-A leading to protein inactivation have also been reported, indicating that the overall glycosylation status of fetuin-A would also be relevant in the regulation of fetuin-A actions [18].

Fetuin-A also has an anti-inflammatory role, acting as endogenous inhibitor of zinc metalloproteases [19]. This feature could explain the association between fetuin-A concentrations and the change in CRP levels over 12 months in the girls receiving SPIOMET, suggesting that fetuin-A levels within the normal range are required to maintain inflammation homeostasis.

The dual functionality of fetuin-A in diabetes risk, inflammation, and cardiovascular disease raises the question as to whether decreasing fetuin-A concentrations should be customarily recommended [20], especially taking into account the heterogeneity of fetuin-A levels reported in different studies using the same method in similar age groups [21].

The finding of lower levels of fetuin-A in PCOS girls was unexpected and could be derived-at least in part-from the status of low-grade inflammation associated with this entity, since it is known that proinflammatory cytokines and proteins such as $\mathrm{CRP}$ - which are increased in PCOS-downregulate fetuin-A expression in the liver $[4,22]$. This would also explain the negative association between fetuin-A concentrations and hepatic fat. SPIOMET treatment was followed by a healthier endocrine-metabolic profile, as judged by the reduction of insulin levels and the drop in ectopic fat; in addition, SPIOMET but not OC raised
fetuin-A levels towards normal. This finding may be perceived as contradictory, because pioglitazone-but not metformin-has been reported to reduce fetuin-A levels in patients with T2D [23]. However, in those studies, pioglitazone was given in monotherapy, and at doses, at least fourfold higher [23].

The main study limitations include the small sample size and the lack of obese PCOS and obese control girls, precluding to discern the separate effects of obesity and PCOS on fetuin-A levels. The strengths include the longitudinal design, the homogeneous study population, and the assessment of the impact of two interventions with divergent effects on the endocrine-metabolic status and, potentially, on cardiometabolic risk. The cross-sectional nature and population heterogeneity of the so far available studies in PCOS women may explain the discrepancies among populations [7-9].

\section{Conclusions}

In conclusion, we report for the first time that fetuin-A levels are reduced in nonobese girls with PCOS and that a low-dose combination of insulin sensitizers and an antiandrogen-but not an OC-increases towards normal fetuin-A concentrations, together with an improvement of the endocrinemetabolic status. The divergent regulation and thus potential duality of fetuin-A effects in diverse pathological conditions deserve further investigation.

\section{Conflicts of Interest}

Marta Díaz, José Miguel Gallego-Escuredo, Abel LópezBermejo, Francis de Zegher, Francesc Villarroya, and Lourdes Ibáñez have no conflicts of interest to disclose. Marta Díaz and Lourdes Ibáñez are clinical investigators of CIBERDEM (Centro de Investigación Biomédica en Red de Diabetes y Enfermedades Metabólicas Asociadas, ISCIII, Madrid, Spain). Francesc Villarroya and José Miguel Gallego-Escuredo are clinical investigators of CIBEROBN (Centro de Investigación Biomédica en Red Fisiopatología de la Obesidad y Nutrición, ISCIII, Madrid, Spain). José Miguel Gallego-Escuredo is a "Sara Borrell" fellow by Instituto de Salud Carlos III, Madrid, Spain. Abel LópezBermejo is a clinical investigator of the I3 Fund for Scientific Research (Ministry of Science and Innovation, Spain). Francis de Zegher is a clinical investigator (Clinical Research Council of Leuven University Hospitals).

\section{Authors' Contributions}

Marta Díaz contributed to study design, researched data, wrote, reviewed, and edited the manuscript. José Miguel Gallego-Escuredo researched the data. Abel López-Bermejo reviewed the manuscript. Francesc Villarroya, Francis de Zegher, and Lourdes Ibáñez contributed to study design and reviewed and edited the manuscript. 


\section{Acknowledgments}

This study was supported by a grant from the ISCIII and the Fondo Europeo de Desarrollo Regional (FEDER), Madrid, Spain (PI15/01078) and by MINECO (SAF2014-55725).

\section{References}

[1] N. Stefan, A. M. Hennige, H. Staiger et al., “ $\alpha_{2}$-HeremansSchmid glycoprotein/ fetuin-A is associated with insulin resistance and fat accumulation in the liver in humans," Diabetes Care, vol. 29, no. 4, pp. 853-857, 2006.

[2] P. R. Srinivas, A. S. Wagner, L. V. Reddy et al., "Serum alpha 2-HS-glycoprotein is an inhibitor of the human insulin receptor at the tyrosine kinase level," Molecular Endocrinology, vol. 7, no. 11, pp. 1445-1455, 1993.

[3] J. H. Ix, M. G. Shlipak, V. M. Brandenburg, S. Ali, M. Ketteler, and M. A. Whooley, "Association between human fetuin-A and the metabolic syndrome: data from the heart and soul study," Circulation, vol. 113, no. 14, pp. 1760-1767, 2006.

[4] S. Sindhu, N. Akhter, S. Shenouda, A. Wilson, and R. Ahmad, "Plasma fetuin-A/ $\alpha 2$-HS-glycoprotein correlates negatively with inflammatory cytokines, chemokines and activation biomarkers in individuals with type-2 diabetes," $B M C$ Immunology, vol. 17, no. 1, pp. 33-33, 2016.

[5] L. Ibáñez, M. Díaz, G. Sebastiani, M. V. Marcos, A. LópezBermejo, and F. de Zegher, "Oral contraception vs insulin sensitization for 18 months in nonobese adolescents with androgen excess: posttreatment differences in C-reactive protein, intima-media thickness, visceral adiposity, insulin sensitivity, and menstrual regularity," The Journal of Clinical Endocrinology \& Metabolism, vol. 98, no. 5, pp. E902-E907, 2013.

[6] M. Díaz, J. M. Gallego-Escuredo, F. de Zegher, F. Villarroya, and L. Ibáñez, "Effects of ethinylestradiol-cyproterone acetate vs. pioglitazone-flutamide-metformin on plasma FGF21 levels in adolescent girls with androgen excess," Diabetes \& Metabolism, vol. 42, no. 3, pp. 196-199, 2016.

[7] R. Abali, C. Celik, N. Tasdemir et al., "The serum protein $\alpha 2$-Heremans-Schmid glycoprotein/fetuin-A concentration and carotid intima-media thickness in women with polycystic ovary syndrome," European Journal of Obstetrics \& Gynecology, and Reproductive Biology, vol. 169, no. 1, pp. 45-49, 2013.

[8] I. Gulhan, G. Bozkaya, D. Oztekin, I. Uyar, A. G. Kebapcilar, and B. Pamuk, "Serum fetuin-A levels in women with polycystic ovary syndrome," Archives of Gynecology and Obstetrics, vol. 286, no. 6, pp. 1473-1476, 2012.

[9] Y. Enli, S. M. Fenkci, V. Fenkci, and O. Oztekin, "Serum fetuin-A levels, insulin resistance and oxidative stress in women with polycystic ovary syndrome," Gynecological Endocrinology, vol. 29, no. 12, pp. 1036-1039, 2013.

[10] L. Ibáñez, L. del Río, M. Díaz et al., "Normalizing ovulation rate by preferential reduction of hepato-visceral fat in adolescent girls with polycystic ovary syndrome," The Journal of Adolescent Health, vol. 61, no. 4, pp. 446-453, 2017.

[11] T. Reinehr and C. L. Roth, "Fetuin-A and its relation to metabolic syndrome and fatty liver disease in obese children before and after weight loss," The Journal of Clinical Endocrinology \& Metabolism, vol. 93, no. 11, pp. 4479-4485, 2008.

[12] A. Ishibashi, Y. Ikeda, T. Ohguro et al., "Serum fetuin-A is an independent marker of insulin resistance in Japanese men,"
Journal of Atherosclerosis and Thrombosis, vol. 17, no. 9, pp. 925-933, 2010.

[13] D. Pal, S. Dasgupta, R. Kundu et al., "Fetuin-A acts as an endogenous ligand of TLR4 to promote lipid-induced insulin resistance," Nature Medicine, vol. 18, no. 8, pp. 1279-1285, 2012.

[14] M. K. Jensen, T. M. Bartz, K. J. Mukamal et al., "Fetuin-A, type 2 diabetes, and risk of cardiovascular disease in older adults: the cardiovascular health study," Diabetes Care, vol. 36, no. 5, pp. 1222-1228, 2013.

[15] M. K. Jensen, T. M. Bartz, L. Djoussé et al., "Genetically elevated fetuin-A levels, fasting glucose levels, and risk of type 2 diabetes: the cardiovascular health study," Diabetes Care, vol. 36, no. 10, pp. 3121-3127, 2013.

[16] M. Sato, Y. Kamada, Y. Takeda et al., "Fetuin-A negatively correlates with liver and vascular fibrosis in nonalcoholic fatty liver disease subjects," Liver International, vol. 35, no. 3, pp. 925-935, 2015.

[17] E. R. Smith, M. L. Ford, L. A. Tomlinson, B. F. Rocks, C. Rajkumar, and S. G. Holt, "Poor agreement between commercial ELISAs for plasma fetuin-A: an effect of protein glycosylation?," Clinica Chimica Acta, vol. 411, no. 17-18, pp. 1367-1370, 2010.

[18] P. M. Karamessinis, A. Malamitsi-Puchner, T. Boutsikou et al., "Marked defects in the expression and glycosylation of $\alpha_{2}$-HS glycoprotein/fetuin-A in plasma from neonates with intrauterine growth restriction: proteomics screening and potential clinical implications," Molecular \& Cellular Proteomics, vol. 7, no. 3, pp. 591-599, 2008.

[19] J. Hedrich, D. Lottaz, K. Meyer et al., "Fetuin-A and cystatin C are endogenous inhibitors of human meprin metalloproteases," Biochemistry, vol. 49, no. 39, pp. 8599-8607, 2010.

[20] A. Berezin, "Is rationale to decrease serum osteoprotegerin and fetuin-A in type 2 diabetes mellitus patients?," Diabetes \& Metabolic Syndrome: Clinical Research \& Reviews, vol. 10, no. 3, pp. 169-170, 2016.

[21] M. Häusler, C. Schäfer, C. Osterwinter, and W. JahnenDechent, "The physiologic development of fetuin-A serum concentrations in children," Pediatric Research, vol. 66, no. 6, pp. 660-664, 2009.

[22] C. Gangneux, M. Daveau, M. Hiron, C. Derambure, J. Papaconstantinou, and J. P. Salier, "The inflammationinduced down-regulation of plasma fetuin-A ( $\alpha 2 \mathrm{HS}-\mathrm{Glyco}-$ protein) in liver results from the loss of interaction between long C/EBP isoforms at two neighbouring binding sites," Nucleic Acids Research, vol. 31, no. 20, pp. 5957-5970, 2003.

[23] A. Esteghamati, M. Afarideh, S. Feyzi, S. Noshad, and M. Nakhjavani, "Comparative effects of metformin and pioglitazone on fetuin-A and osteoprotegerin concentrations in patients with newly diagnosed diabetes: a randomized clinical trial," Diabetes \& Metabolic Syndrome: Clinical Research \& Reviews, vol. 9, no. 4, pp. 258-265, 2015. 


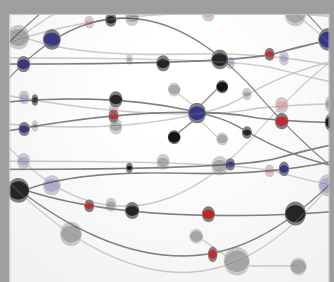

The Scientific World Journal
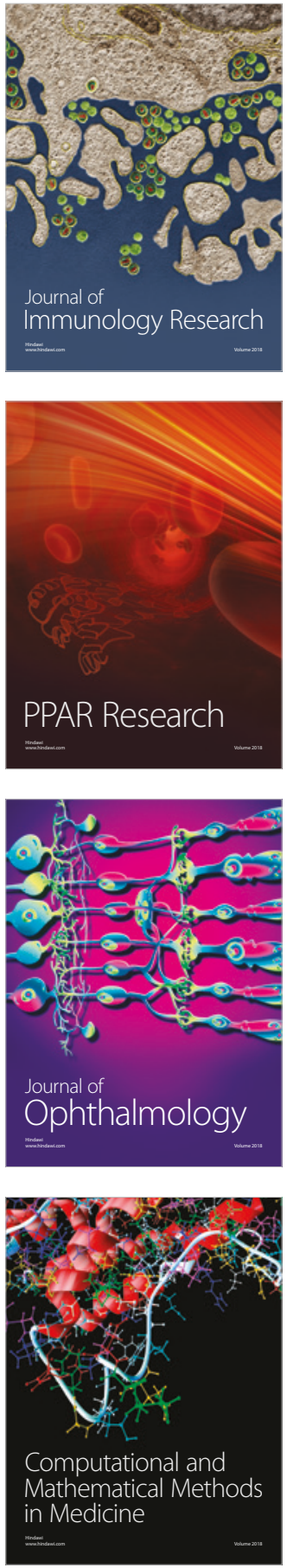

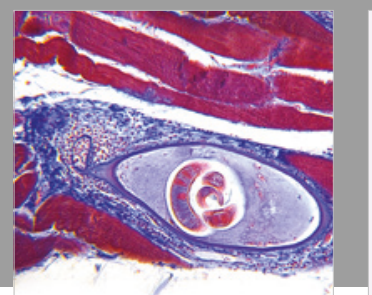

Gastroenterology Research and Practice

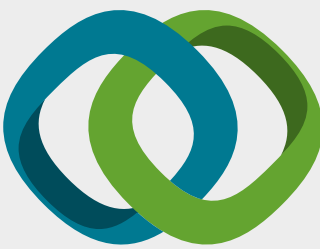

\section{Hindawi}

Submit your manuscripts at

www.hindawi.com
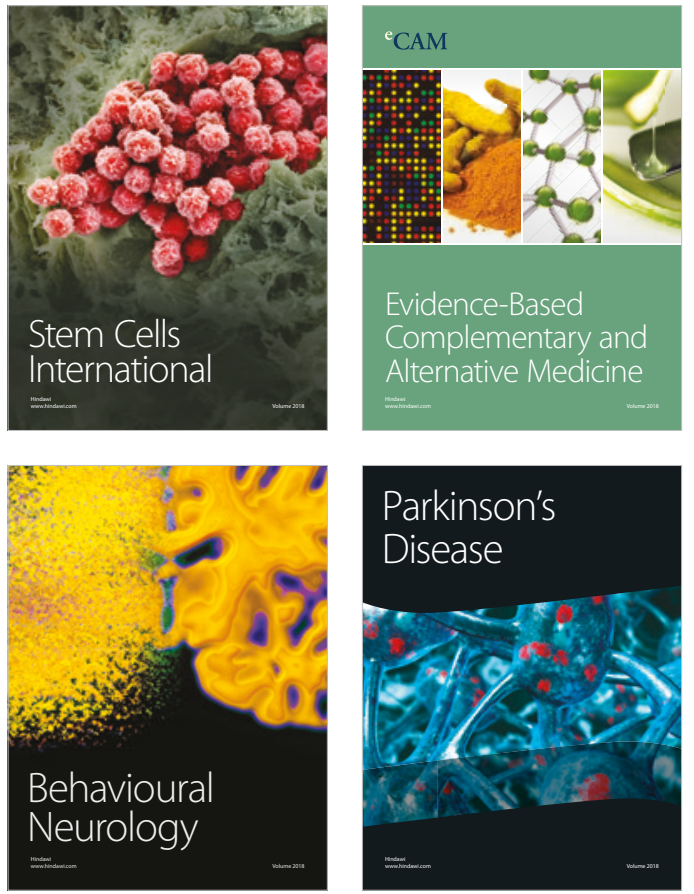

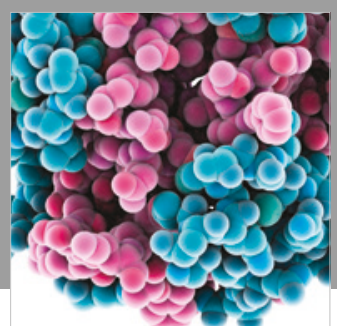

ournal of

Diabetes Research

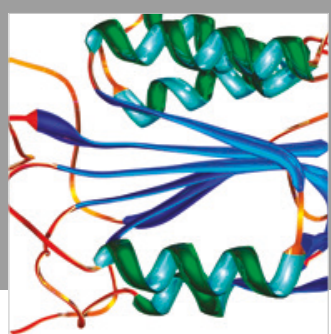

Disease Markers
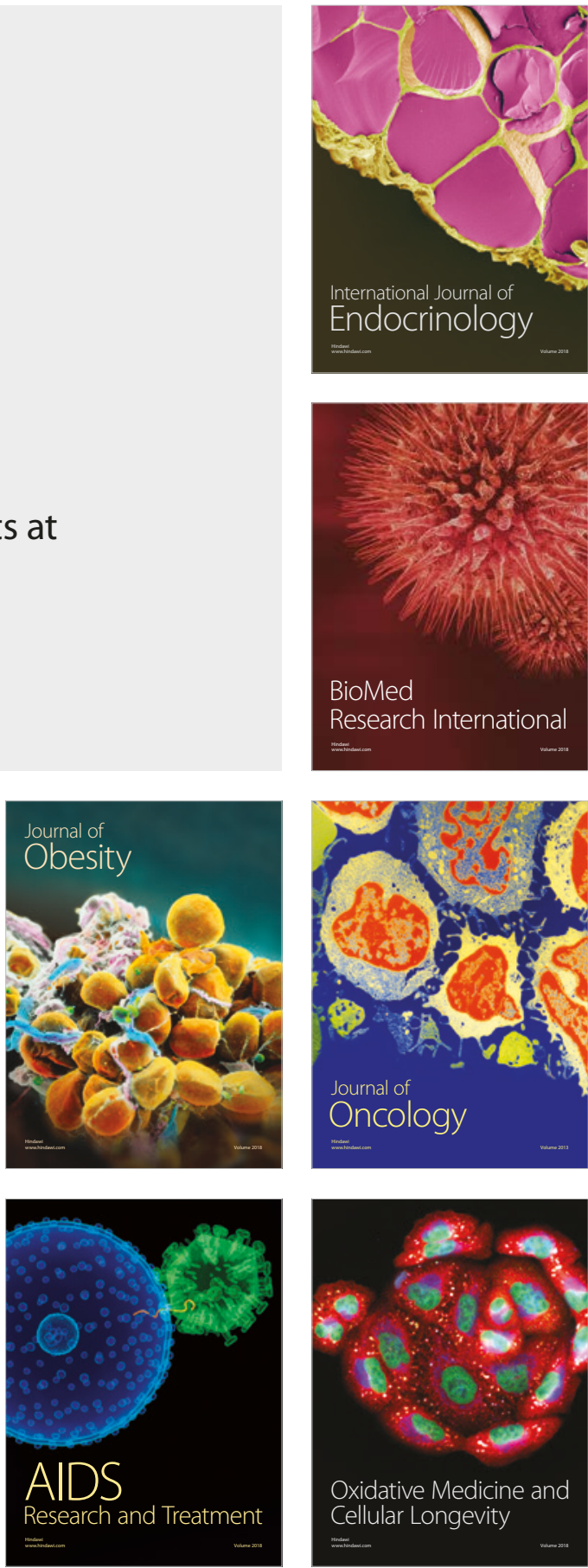